\title{
Time-Dependent Density Functional Theory Beyond the Adiabatic Local Density Approximation
}

\author{
G. Vignale and C. A. Ullrich \\ Department of Physics, University of Missouri, Columbia, Missouri 65211 \\ S. Conti \\ Scuola Normale Superiore, I-56126 Pisa, Italy
}

(Received 27 June 1997)

\begin{abstract}
In the current density functional theory of linear and nonlinear time-dependent phenomena, the treatment of exchange and correlation beyond the level of the adiabatic local density approximation is shown to lead to the appearance of viscoelastic stresses in the electron fluid. Complex and frequency-dependent viscosity/elasticity coefficients are microscopically derived and expressed in terms of properties of the homogeneous electron gas. As a first consequence of this formalism, we provide an explicit formula for the linewidths of collective excitations in electronic systems. [S0031-9007(97)04789-3]
\end{abstract}

PACS numbers: 71.45.Gm, 21.10.Re, 73.20.Dx, 78.30.Fs

Time-dependent density functional theory (TDFT) [1] is frequently invoked as a tool for studying the dynamics of many-particle systems. This theory maps the difficult problem of interacting electrons in a time-dependent external potential $V(\vec{r}, t)$ to the simpler one of noninteracting electrons in an effective time-dependent potential $V_{\text {eff }}(\vec{r}, t)=V(\vec{r}, t)+v_{H}(\vec{r}, t)+v_{\mathrm{xc}}(\vec{r}, t)$ [where $v_{H}$ is the Hartree potential, and $v_{\mathrm{xc}}$ is the exchange-correlation (xc) potential] yielding the same density $n(\vec{r}, t)$. In order to obtain a practical computational scheme, the xc potential is usually approximated as a function of the instantaneous local density,

$$
v_{\mathrm{xc}}^{\mathrm{ALDA}}(n(\vec{r}, t))=\left(\frac{d \epsilon_{\mathrm{xc}}(n)}{d n}\right)_{n=n(\vec{r}, t)},
$$

where $\epsilon_{\mathrm{xc}}(n)$ is the xc energy density of the homogeneous electron gas of density $n$. This scheme is known as the "adiabatic local density approximation" (ALDA) [2]. Because the conditions of validity of the ALDA (slowly varying density and potential in time as well as in space) are seldom met in experiments, a few attempts have been made $[3,4]$ to improve upon the ALDA. The objective of these attempts was to obtain approximations for the $\mathrm{xc}$ potential which would still be local in space, but not in time. All these approximations were found to suffer from inconsistencies, such as the failure to satisfy the so called "harmonic potential theorem" (HPT) [4-6], or other basic symmetries. Only recently, it has become clear that the root of these difficulties lies in the fact that the xc potential in TDFT is an intrinsically nonlocal functional of the density, that is, a functional that does not admit a gradient expansion [7,8].

Fortunately, a local gradient expansion is still possible if the theory is formulated in terms of the current density. This was shown in Ref. [7] by Vignale and Kohn, who developed the time-dependent current density functional approach to the linear response theory, and gave an explicit expression for the linearized xc vector potential $\vec{a}_{\mathrm{xc}}(\vec{r}, \omega)$ for a system of slowly varying density, subject to a spatially slowly varying external vector potential at a finite frequency $\omega$. Their expression becomes exact in the limit $k \ll \omega / v_{\mathrm{F}}, k_{\mathrm{F}}$ and $q \ll \omega / v_{\mathrm{F}}, k_{\mathrm{F}}$, where $k^{-1}$ and $q^{-1}$ are the characteristic length scales for variation of the external potential and equilibrium density, respectively, and $k_{\mathrm{F}}$ and $v_{\mathrm{F}}$ are the local Fermi momentum and velocity. However, the final expression for $\vec{a}_{\mathrm{xc}}(\vec{r}, \omega)$ in Ref. [7] is rather formidable, and its physical meaning is far from transparent. Furthermore, it is restricted to the linear response regime. It is the purpose of this paper to overcome these limitations.

In the following we derive a consistent local theory of the nonlinear dynamical response of a quantum electronic system of "slowly varying density", in the sense specified above. The effect of the xc potential beyond the ALDA will be shown to be analogous to the introduction of viscoelastic stresses in classical fluid dynamics and elasticity theory [9]. The generalized viscosity coefficients (or, equivalently, the generalized bulk and shear moduli) are complex and frequency-dependent functions of the density, and can be calculated in terms of the local field factors of the uniform electron gas [10,11]. An important consequence of the xc viscosity is to provide a damping mechanism for long-lived collective excitations which cannot efficiently decay into particle-hole pairs - the only form of damping allowed within the ALDA [12].

We begin by recasting the linear response theory of $[7,8]$ in a form that is suitable for the nonlinear generalization. Let $n_{0}(\vec{r})$ be the ground-state density of the system, and let $\chi_{\mathrm{KS}, i j}\left(\vec{r}, \vec{r}^{\prime}, \omega\right)$ be the currentcurrent response function of a system of noninteracting electrons whose ground-state density is also $n_{0}(\vec{r})$. The system is perturbed by a weak external vector potential $\vec{a}_{1}(\vec{r}, t)=\vec{a}_{1}(\vec{r}, \omega) e^{-i \omega t}[13]$, and we want to calculate 
the amplitude of the current-density response $\vec{j}_{1}(\vec{r}, t)=$ $\vec{j}_{1}(\vec{r}, \omega) e^{-i \omega t}$, to first order in $\vec{a}_{1}$. The answer is

$$
\begin{aligned}
j_{1, i}(\vec{r}, \omega)= & \int \sum_{j} \chi_{\mathrm{KS}, i j}\left(\vec{r}, \vec{r}^{\prime}, \omega\right)\left[a_{1, j}\left(\vec{r}^{\prime}, \omega\right)\right. \\
& \left.+a_{\mathrm{H} 1, j}\left(\vec{r}^{\prime}, \omega\right)+a_{\mathrm{xc} 1, j}\left(\vec{r}^{\prime}, \omega\right)\right] d \vec{r}^{\prime},
\end{aligned}
$$

where $\vec{a}_{\mathrm{H} 1}$ is the first-order change in the Hartree potential (written in vector-potential form [13]), and $\vec{a}_{\mathrm{xc} 1}$ is the first-order xc vector potential, which contains the manybody effects. Note that $\vec{a}_{\mathrm{xc} 1}$ in general has both longitudinal and transverse components, even when $\vec{a}_{1}$ is purely longitudinal. The local-density approximation for $\vec{a}_{\mathrm{xc} 1}$ was first derived in Ref. [7], Eq. (19). We have found that that complicated formula can be written in a physically transparent form. To this end, we introduce the xc "electric field" $\vec{E}_{\mathrm{xc} 1}(\vec{r}, \omega) \equiv \frac{i \omega}{c} \vec{a}_{\mathrm{xc} 1}(\vec{r}, \omega)$. Then

$$
\begin{aligned}
-e E_{\mathrm{xc} 1, i}(\vec{r}, \omega)= & -\vec{\nabla}_{i} v_{\mathrm{xc} 1}^{\mathrm{ALDA}}(\vec{r}, \omega) \\
& +\frac{1}{n_{0}(\vec{r})} \sum_{j} \frac{\partial \sigma_{\mathrm{xc}, i j}(\vec{r}, \omega)}{\partial r_{j}}
\end{aligned}
$$

( $e$ is the absolute value of the electron charge, and $c$ is the speed of light). The first term is the linearization of the ALDA expression (1), and the dynamical correction is the divergence of the viscoelastic stress tensor

$$
\begin{aligned}
\sigma_{\mathrm{xc}, i j}= & \tilde{\eta}_{\mathrm{xc}}\left(\frac{\partial u_{i}}{\partial r_{j}}+\frac{\partial u_{j}}{\partial r_{i}}-\frac{2}{3} \vec{\nabla} \cdot \vec{u} \delta_{i j}\right) \\
& +\tilde{\zeta}_{\mathrm{xc}} \vec{\nabla} \cdot \vec{u} \delta_{i j} .
\end{aligned}
$$

Here $\vec{u}(\vec{r}, \omega) \equiv \vec{j}_{1}(\vec{r}, \omega) / n_{0}(\vec{r})$ is the velocity field, and $\vec{\eta}_{\mathrm{xc}}\left[\omega, n_{0}(\vec{r})\right]$ and $\tilde{\zeta}_{\mathrm{xc}}\left[\omega, n_{0}(\vec{r})\right]$ are complex viscosity coefficients. They are related to the homogeneous electron gas functions $f_{\mathrm{xc}, L}^{h}(\omega, n)$ and $f_{\mathrm{xc}, T}^{h}(\omega, n)$ ( $L$ for longitudinal, $T$ for transverse) used in Ref. [7] as follows:

$$
\tilde{\zeta}_{\mathrm{xc}}(\omega, n)=-\frac{n^{2}}{i \omega}\left[f_{\mathrm{xc}, L}^{h}-\frac{4}{3} f_{\mathrm{xc}, T}^{h}-\frac{d^{2} \epsilon_{\mathrm{xc}}(n)}{d n^{2}}\right]
$$

and

$$
\tilde{\eta}_{\mathrm{xc}}(\omega, n)=-\frac{n^{2}}{i \omega} f_{\mathrm{xc}, T}^{h} .
$$

The functions $f_{\mathrm{xc}, L(T)}^{h}(\omega, n)$ are defined in terms of the dynamical local field factors $G_{L(T)}(k, \omega)$ [14] as $f_{\mathrm{xc}, L(T)}^{h}(\omega, n) \equiv-\lim _{k \rightarrow 0} 4 \pi e^{2} G_{L(T)}(k, \omega) / k^{2}$. They have recently been calculated, within a mode-coupling approximation scheme, at zero temperature, by Conti et al. $[10,11]$. Because the local field factors are singular for small $k$ and $\omega$, it is important to keep track of the order of the limits $k \rightarrow 0$ and $\omega \rightarrow 0$. The $f_{\mathrm{xc}}^{h}$ 's are defined by taking the limit $k \rightarrow 0$ first. Thus the limit for $\omega \rightarrow 0$ of $f_{\mathrm{xc}, L}^{h}(\omega, n)$ differs from the familiar $\lim _{k \rightarrow 0} 4 \pi e^{2} G_{L}(k, 0) / k^{2}=-d^{2} \epsilon_{\mathrm{xc}}(n) / d n^{2} . \quad$ However, rigorous low frequency limits [15] can be obtained [16] from an analysis of the transport equation in the Landau theory of Fermi liquids [17]. We find

$$
\lim _{\omega \rightarrow 0} \operatorname{Re}\left[f_{\mathrm{xc}, L}^{h}(\omega, n)-\frac{4}{3} f_{\mathrm{xc}, T}^{h}(\omega, n)-\frac{d^{2} \epsilon_{\mathrm{xc}}(n)}{d n^{2}}=0\right.
$$

and

$$
\lim _{\omega \rightarrow 0} \operatorname{Re} f_{\mathrm{xc}, T}^{h}(\omega, n)=\frac{2 E_{F}}{5 n} \frac{F_{2} / 5-F_{1} / 3}{1+F_{1} / 3},
$$

where $F_{0}, F_{1}, \ldots$ are the usual dimensionless Landau parameters of the homoegeneous electron gas [17]. As for the imaginary parts, one finds $\operatorname{Im} f_{\mathrm{xc}, L(T)}^{h} \sim-c_{0, L(T)} \omega$ for $\omega \rightarrow 0$, where the approximate values of the coefficients $c_{0, L(T)}$ are tabulated in Ref. [11].

From these results, one concludes that the real parts of the xc viscosity coefficients (which agree with the ordinary notion of fluid viscosities) have finite values in the limit $\omega \rightarrow 0$. The imaginary parts of the viscosity coefficients are better understood in terms of bulk and shear moduli of an isotropic elastic medium, $K_{\mathrm{xc}}^{\mathrm{dyn}}$ and $\mu_{\mathrm{xc}}^{\mathrm{dyn}}$. According to elasticity theory [9], we define $K_{\mathrm{xc}}^{\mathrm{dyn}}(\omega)=\omega \operatorname{lm} \tilde{\zeta}_{\mathrm{xc}}$ and $\mu_{\mathrm{xc}}^{\mathrm{dyn}}(\omega)=\omega \operatorname{Im} \tilde{\eta}_{\mathrm{xc}}$. The superscript $d y n$ is a reminder that these are dynamical contributions to be added to the usual static ones, already present in the ALDA. The static elastic constants are $K_{\mathrm{xc}}^{\text {stat }}=n^{2} d^{2} \epsilon_{\mathrm{xc}}(n) / d n^{2}$ and $\mu_{\mathrm{xc}}^{\text {stat }}=0$, respectively. Equations (7) and (8) show that for $\omega \rightarrow 0 K_{\mathrm{xc}}^{\mathrm{dyn}}$ vanishes, while $\mu_{\mathrm{xc}}^{\mathrm{dyn}}$ has a finite value. A similiar state of affairs holds for the noninteracting kinetic contributions to the bulk and shear moduli: $K_{\mathrm{kin}}^{\mathrm{dyn}}=0$ and $\mu_{\mathrm{kin}}^{\mathrm{dyn}}=p(n)$, where $p(n)$ is the noninteracting Fermi pressure (see below), and $K_{\mathrm{kin}}^{\text {stat }}=n d p(n) / d n, \mu_{\mathrm{kin}}^{\text {stat }}=0 . \quad$ The general conclusion is that dynamical (post-ALDA) effects do not modify the bulk modulus, but they cause the appearance of a nonvanishing shear modulus and viscosity.

Equations (3) and (4) clearly display the basic symmetries which were used in the derivation of $[7,8]$. First of all, the fact that the force exerted by the xc potential per unit volume $-e n_{0}(\vec{r}) \vec{E}_{\mathrm{xc} 1}(\vec{r}, \omega)-n_{1}(\vec{r}, \omega) \vec{\nabla} v_{\mathrm{xc} 0}(\vec{r})$ can be written as the divergence of a symmetric rank 2 local tensor guarantees that the net force and the net torque acting on a volume element of the fluid have no contribution from the volume element itself (Newton's third law). Therefore, the HPT, the "zero-force" and "zero-torque" theorems of $[7,8]$ are manifestly satisfied. (Note that the force exerted by the xc "magnetic field" $\vec{B}_{\mathrm{xc}}=\vec{\nabla} \times \vec{a}_{\mathrm{xc}}$ is legitimately disregarded in this argument, being of higher order in the gradient expansion [see also below]. Besides, it is rigorously absent in the linear response theory, if there is no static magnetic field).

Thus far, we have used the condition of slow density variation $\left(k, q \ll \omega / v_{\mathrm{F}}\right)$ only in approximating the $\mathrm{xc}$ vector potential. If this condition is met in the physical system under study, we can also use it to approximate the Kohn-Sham (KS) response function, $\chi_{\mathrm{KS}, i j}\left(\vec{r}, \vec{r}^{\prime}, \omega\right)$. 
Then Eq. (2) for the current reduces (after considerable algebraic manipulation) to a linearized Navier-Stokes equa- tion [9] with complex and frequency-dependent viscosity coefficients:

$$
-i m \omega j_{1, i}(\vec{r}, \omega)=n_{0}(\vec{r})\left[-i \omega \frac{e}{c} a_{i}(\vec{r}, \omega)-\nabla_{i}\left(\frac{p_{1}(n)}{n_{0}}+v_{\mathrm{H} 1}(\vec{r}, \omega)+v_{\mathrm{xc} 1}^{\mathrm{ALDA}}(\vec{r}, \omega)\right)\right]+\sum_{j} \frac{\partial \sigma_{i j}(\vec{r}, \omega)}{\partial r_{j}}
$$

Here $p_{1}(n)=p(n)-p\left(n_{0}\right)$ is the first order change in the pressure of the noninteracting electron fluid $[p(n)=$ $\left.\left(3 \pi^{2}\right)^{2 / 3} \hbar^{2} n^{5 / 3} / 5 m\right]$, and $v_{\mathrm{H} 1}, v_{\mathrm{xc} 1}^{\mathrm{ALA}}$ are the first-order changes in the Hartree potential and ALDA xc potential. The full stress tensor $\sigma_{i j}$ is defined as in Eq. (4), with the viscosity coefficient $\tilde{\eta}_{\mathrm{xc}}$ replaced by $\tilde{\eta}_{\mathrm{xc}}-p\left(n_{0}\right) / i \omega$, while $\tilde{\zeta}_{x c}$ remains unchanged.

If, on the other hand, the conditions $k, q \ll \omega / v_{F}$ are not well satisfied by our system, then it is better to revert to the original KS formulation (2), which treats the noninteracting response exactly. The use of the local density approximation (3) for the xc potential becomes then an uncontrolled approximation, but it may still work well in practice. In particular, we note that Eq. (2) allows for the phenomenon of Landau damping (damping of collective modes by single electron-hole pairs), while Eq. (9) does not.

Let us now discuss the generalization of the formalism to the nonlinear response regime. In this case, one must solve the full time-dependent $\mathrm{KS}$ equations for the KS orbitals $\psi_{\alpha}(\vec{r}, t)$

$$
\begin{gathered}
{\left[i \hbar \frac{\partial}{\partial t}-\frac{1}{2 m}\left(-i \hbar \vec{\nabla}+\frac{e}{c} \vec{a}(\vec{r}, t)+\frac{e}{c} \vec{a}_{\mathrm{xc}}(\vec{r}, t)\right)^{2}-\right.} \\
\left.v_{0}(\vec{r})-v_{H}(\vec{r}, t)\right] \psi_{\alpha}(\vec{r}, t)=0,
\end{gathered}
$$

starting, for example, with the static KS orbitals corresponding to the external potential $v_{0}(\vec{r})$ at the initial time.

The density and the current density are computed from the KS orbitals according to the usual rules [3]. The form of the nonlinear xc vector potential is dictated by the following requirements: (i) the $\mathrm{xc}$ force density

$$
F_{\mathrm{xc}, i}=n \frac{e}{c}\left[\left(\frac{\partial}{\partial t}+\vec{u} \cdot \vec{\nabla}\right) a_{\mathrm{xc}, i}-\sum_{k} u_{k} \nabla_{i} a_{\mathrm{xc}, k}\right]
$$

must be the divergence of a local symmetric rank two stress tensor (Newton's third law). "Locality" here means that $\sigma_{\mathrm{xc}, i j}(\vec{r}, t)$ is a function of $n\left(\vec{R}, t^{\prime}\right), \vec{j}\left(\vec{R}, t^{\prime}\right)$, and their spatial derivatives, where $t^{\prime}<t$, and $\vec{R}\left(t^{\prime} \mid \vec{r}, t\right)$ is the position at time $t^{\prime}$ of the fluid element which evolves into $\vec{r}$ at time $t$ [18]. (ii) Under transformation to an accelerated frame of reference [5] with origin at $\vec{x}(t)$, the stress tensor $\sigma_{\mathrm{xc}, i j}(\vec{r}, t)$ becomes $\sigma_{\mathrm{xc}, i j}^{\prime}(\vec{r}, t)=\sigma_{\mathrm{xc}, i j}(\vec{r}+\vec{x}(t), t)$. (iii) Equation (10) must reduce to the linear response theory in the limit of small external perturbations, and to the nonlinear Navier-Stokes equation in the limit of slowly varying (in time) perturbation [19].

To within the accuracy of our approximation, i.e., to second order in the spatial derivatives, the above requirements uniquely determine the form of $\vec{a}_{\mathrm{xc}}$ :

$$
\begin{aligned}
\frac{e}{c} \frac{\partial a_{\mathrm{xc}, i}(\vec{r}, t)}{\partial t}= & -\nabla_{i} v_{\mathrm{xc}}^{\mathrm{ALDA}}(\vec{r}, t) \\
& +\frac{1}{n(\vec{r}, t)} \sum_{j} \frac{\partial \sigma_{\mathrm{xc}, i j}(\vec{r}, t)}{\partial r_{j}},
\end{aligned}
$$

where

$$
\begin{gathered}
\sigma_{\mathrm{xc}, i j}(\vec{r}, t)=\int_{-\infty}^{t}\left\{\tilde{\eta}\left(n(\vec{r}, t), t-t^{\prime}\right)\left[\frac{\partial u_{i}\left(\vec{r}, t^{\prime}\right)}{\partial r_{j}}+\frac{\partial u_{j}\left(\vec{r}, t^{\prime}\right)}{\partial r_{i}}-\frac{2}{3} \vec{\nabla} \cdot \vec{u}\left(\vec{r}, t^{\prime}\right) \delta_{i j}\right]\right. \\
\left.+\tilde{\zeta}\left(n(\vec{r}, t), t-t^{\prime}\right) \vec{\nabla} \cdot \vec{u}\left(\vec{r}, t^{\prime}\right) \delta_{i j}\right\} d t^{\prime},
\end{gathered}
$$

$\tilde{\eta}\left(n, t-t^{\prime}\right) \equiv \int \tilde{\eta}(n, \omega) \exp \left[-i \omega\left(t-t^{\prime}\right)\right] d \omega / 2 \pi$, and similarly for $\tilde{\zeta}$. Here $n(\vec{r}, t)$ and $\vec{u}(\vec{r}, t)$ are the timedependent values of the density and velocity field.

Note that our formula for $\vec{a}_{\mathrm{xc}}$ is still linear in $\vec{u}(\vec{r}, t)$. This happens because, due to the constraint (ii) of generalized Galilean invariance, the velocity must enter the stress tensor through its spatial derivatives, which are assumed to be small, even if the velocity itself is not small. Terms of higher order in the velocity would necessarily be of higher order in the gradient expression. For the same reason, one can ignore the velocity-dependent terms in the xc force [Eq. (11)], and the difference between $\vec{r}$ and the "retarded position" $\vec{R}$ of the fluid element. Similarly, the apparent ambiguity of whether the density entering the viscosity coefficients in Eq. (13) should be evalu- ated at time $t$ or at some earlier time $t^{\prime}$, is resolved by noting that the difference $n\left(\vec{r}, t^{\prime}\right)-n(\vec{r}, t)=\int_{t^{\prime}}^{t} \vec{\nabla}$. $\vec{j}(\vec{r}, \tau) d \tau$ generates a higher order gradient correction.

The simple form of Eq. (12) is justified by our basic assumption that the gradients of the density and velocity fields be small. By using the full expression (11) for the electromagnetic force on the left hand side of Eq. (12), and by replacing $\left(\vec{r}, t^{\prime}\right) \rightarrow\left[\vec{R}\left(t^{\prime} \mid \vec{r}, t\right), t^{\prime}\right]$ on the right hand side of Eq. (13), the approximation can be systematically improved, so as to satisfy the local "zero-force" theorem to all orders in the gradients. A straightforward generalization allows one to construct an approximation that also satisfies the local "zero-torque" theorem.

Finally, we wish to comment on the condition $k, q \ll \omega / v_{\mathrm{F}}$ which defines the limit of validity of our 
approximate treatment of $\vec{a}_{\mathrm{xc}}$. This restriction is forced on us by the analytic structure of the functions $f_{\mathrm{xc} L(T)}^{h}(k, \omega)$ which are singular along the line $\omega=k v_{\mathrm{F}}$, thus limiting the radius of convergence of the small- $k$ expansion. The condition is reasonably well satisfied at the characteristic frequencies of collective excitations, but it becomes increasingly restrictive as we lower the frequency.

Let us now briefly discuss an application of the formalism developed above. It is well known that high-frequency collective excitations which are not efficiently Landau damped would appear as sharp $\delta$ functions within the ALDA. In this regime, the only efficient damping mechanism, leading to a nonvanishing linewidth, is provided by the dynamical xc effects. Making again use of the analogy with classical hydrodynamics [9], we can calculate the linewidth (twice the imaginary part of the frequency) as $\Gamma=\left|\bar{E}^{\prime}\right| / \bar{E}$, where $\bar{E}^{\prime}=2 \operatorname{Re} \sum_{i, k} \int d \vec{r} u_{i}^{*}(\vec{r}, \omega) \nabla_{k} \sigma_{\mathrm{xc}, i k}(\vec{r}, \omega)$ is the average energy dissipated per unit time by the viscosity, and $\vec{E}=2 m \int d \vec{r} n_{0}(\vec{r})|\vec{u}(\vec{r}, \omega)|^{2}$ is the mechanical energy stored in the oscillation, regarded as slowly decaying in time. Thus,

$$
\Gamma=\frac{\left|\operatorname{Re} \sum_{i, k} \int d \vec{r} u_{i}^{*}(\vec{r}, \omega) \nabla_{k} \sigma_{\mathrm{xc}, i k}(\vec{r}, \omega)\right|}{m \int d \vec{r} n_{0}(\vec{r})|\vec{u}(\vec{r}, \omega)|^{2}},
$$

where $\vec{u}(\vec{r}, \omega)$ is the velocity field of the collective mode calculated within the ALDA, and $n_{0}(\vec{r})$ is the equilibrium density. This classical result is confirmed by a full quantum mechanical calculation of the poles of the current-current response function. Plasmon oscillations in low-impurity quantum wells appear to be a promising ground for the application of Eq. (14), since in these systems the density is slowly varying and band structure effects are negligible. To obtain an order of magnitude estimate of the xc linewidth for plasmons of small wave vector $k$, we consider the homogeneous case where Eq. (14) reduces to the known result [14] $\Gamma / \omega_{p}=$ $\left(n_{0} k^{2} / m \omega_{p}^{2}\right)\left|\operatorname{Im} f_{\mathrm{xc} L}^{h}\left(n_{0}, \omega_{p}\right)\right|$. This limit can be experimentally realized in wide parabolic quantum wells. Using results of Ref. [11] for $f_{\mathrm{xcL}}^{h}$ in three dimensions, we get $\Gamma / \omega_{p}=0.027\left(k / k_{\mathrm{F}}\right)^{2}$ for a typical $r_{s}=4$. This is about an order of magnitude smaller than the linewidth observed for plasmons in simple metals [20], where also the band structure contributes. Detailed applications of Eq. (14) to quantum wells will be reported elsewhere.

This work was supported by NSF Grant No. DMR9403908 and by a Research Board Grant from the University of Missouri. S. C. acknowledges support from a traveling scholarship from Scuola Normale Superiore. We acknowledge useful discussions with Walter Kohn, Hardy Gross, John Dobson, and Mario Tosi.

[1] E. Runge and E. K. U. Gross, Phys. Rev. Lett. 52, 997
(1984).

[2] A. Zangwill and P. Soven, Phys. Rev. Lett. 45, 204 (1980); Phys. Rev. B 24, 4121 (1981).

[3] E. K. U. Gross and W. Kohn, Phys. Rev. Lett. 55, 2850 (1985); 57, 923(E) (1986). See also E. K. U. Gross, J.F. Dobson, and M. Petersilka, in Topics in Current Chemistry, edited by R.F. Nalewajski (Springer, Berlin, 1996), Chap. 2.

[4] J. Dobson, Phys. Rev. Lett. 73, 2244 (1994); in Density Functional Theory, edited by E. K. U. Gross and R.M. Dreizler (Plenum, New York, 1994), p. 393.

[5] G. Vignale, Phys. Rev. Lett. 74, 3233 (1995).

[6] W. Kohn, Phys. Rev. 123, 1242 (1961); L. Brey et al., Phys. Rev. B 40, 10647 (1989); 42, 1240 (1990); S. K. Yip, Phys. Rev. B 43, 1707 (1991).

[7] G. Vignale and W. Kohn, Phys. Rev. Lett. 77, 2037 (1996).

[8] G. Vignale and W. Kohn, in Electronic Density Functional Theory, edited by J. Dobson, M.K. Das, and G. Vignale (Plenum Press, New York, 1996).

[9] L. D. Landau and E. Lifshitz, Mechanics of Fluids, Course of Theoretical Physics Vol. 6 (Pergamon Press, Oxford, 1987), 2nd ed; Theory of Elasticity, Course of Theoretical Physics Vol. 7 (Pergamon Press, Oxford, 1986), 3rd ed.

[10] H. M. Böhm, S. Conti, and M. P. Tosi, J. Phys. Condens. Matter 8, 781 (1996).

[11] S. Conti, R. Nifosì, and M. P. Tosi, J. Phys. Condens. Matter 9, L475 (1997).

[12] The connection between hydrodynamics and linear response theory was described by Leo P. Kadanoff and P. C. Martin, Ann. Phys. 24, 419 (1963). For a recent review of the application of hydrodynamics to electronic systems, see E. Zaremba and H.C. Tso, Phys. Rev. B 49, 8147 (1994), and references therein.

[13] We work in a gauge in which the dynamical external scalar potential is zero. In general, a scalar potential $V(\vec{r}, t)$ is equivalent, by gauge invariance, to a longitudinal vector potential $\vec{a}(\vec{r}, t)=c \int^{t} \vec{\nabla} V(\vec{r}, \tau) d \tau$.

[14] K. S. Singwi and M. P. Tosi, in Solid State Physics, edited by H. Ehrenreich, F. Seitz, and D. Turnbull (Academic, New York, 1981), Vol. 36, p. 177.

[15] The limit $\omega \rightarrow 0$ here means $\omega \ll E_{\mathrm{F}}$ while the condition $\omega / v_{\mathrm{F}} \gg k, q$ is still satisfied. At finite temperature $T$, we must also require $\omega \gg 1 / \tau$ where $\tau \sim T^{-2}$ is the collisional relaxation time. Similarly, if $\tau_{\text {ext }}$ is the relaxation time due to collisions with external impurities, we require $\omega \gg 1 / \tau_{\text {ext }}$.

[16] S. Conti and G. Vignale (to be published).

[17] D. Pines and P. Nozières, The Theory of Quantum Liquids (W. A. Benjamin, New York, 1966), Chap. 1.

[18] The importance of the "retarded position" in this context has first been pointed out by J.F. Dobson, M. J. Bünner, and E. K. U. Gross, Phys. Rev. Lett. 79, 1905 (1997).

[19] The connection between the time-dependent Schrödinger equation and hydrodynamics is discussed, for example, in B. M. Deb and S. K. Ghosh, J. Chem. Phys. 77, 342 (1982); M. Himi and K. Fukushima, Nucl. Phys. A431, 161 (1984). See also Ref. [12] above.

[20] A. vom Felde, J. Sprösser-Prou, and J. Fink, Phys. Rev. B 40, 10181 (1989). 